\title{
¿Es tan necesario cambiar nuestro sistema publico de salud?
}

\author{
Is it so necessary to change our public Health System?
}

\section{Antonio Pose Reino}

Jefe Servicio Medicina Interna. Complexo hospitalario universitario de Santiago de Compostela. Profesor Titular de Medicina. Universidad de Santiago de Compostela

En los próximos 8 años se espera un cambio demográfico poblacional similar al ocurrido en los últimos 40 años, donde el $30 \%$ de la población será mayor de 65 años. El 30\% de esta población mayor de 65 años se considera frágil ${ }^{1}$. Si bien es cierto que fragilidad no es lo mismo que comorbilidad o multimorbilidad, sí que se relacionan o al menos coinciden en múltiples ocasiones y podemos decir que la tercera parte de la población adulta y especialmente de los mayores de 65 años, tienen una enfermedad crónica.

Los cambios demográficos ocurridos en los últimos años nos han llevado a un aumento espectacular del gasto sanitario y han hecho que nuestro sistema sanitario público se encuentre en muchas ocasiones saturado y no pueda atender de forma adecuada las necesidades de la población. El sistema sanitario actual maneja al paciente crónico como si fuera un paciente agudo, y este es el motivo principal por el que ha fracasado. El paciente agudo se trata durante el episodio y después no precisa seguimiento, pero el paciente crónico se beneficia del tratamiento de los brotes agudos de su enfermedad, y también del seguimiento posterior, lo que servirá para detectar las descompensaciones de forma precoz y evitar en muchos casos el ingreso hospitalario por este motivo, mejorando el pronóstico, su situación funcional y su calidad de vida. Si tenemos en cuenta que cerca del 30\% de nuestra población tiene una enfermedad crónica, seremos conscientes de que tenemos que cambiar el enfoque y nuestra estrategia; si queremos seguir manteniendo un sistema público de salud tan bueno y tan eficiente como el que teníamos.

En la actualidad, sabemos que el $5 \%$ de la población consume el $50 \%$ de los recursos y se hace necesario detectar cuales son esos pacientes para actuar sobre ellos de forma proactiva, pero siendo conscientes de que una actuación dirigida sólo a este pequeño subgrupo de la población no será suficiente y precisamos actuar sobre estratos más bajos de la población, incluyendo los crónicos que aún no consumen tantos recursos y que funcionalmente están mejor, pero que tarde o temprano subirán al último escalón de la pirámide poblacional, y esto es los que hay que conseguir que sea lo más tarde posible, manteniendo la máxima funcionalidad y tratando de evitar ingresos y descompensaciones que irán mermando su funcionalidad hasta hacerlos crónicos complejos. Existen diferentes modelos para estratificar a la población, pero está claro que es necesario hacerlo. Esto se puede hacer desde arriba hacia abajo, con un criterio socioeconómico y de gasto a través de la administración o hacerlo desde abajo hacia arriba, a partir de los clínicos. Estamos convencidos de que es esta última, la forma que mejor va a identificar a aquellos pacientes que se van a beneficiar de una atención más personalizada ya sea sanitaria, social, activa o paliativa.

Otro aspecto importante en el nuevo modelo de sistema de salud es la implicación del paciente. Es necesario formar al paciente portador de una enfermedad crónica para que el mismo sea capaz de tomar decisiones compartidas sobre el manejo de su enfermedad, lo que conocemos como el "empoderamiento". La tecnología ha evolucionado de forma espectacular en los últimos años y también su aplicación a la medicina, esto ha permitido mejorar las técnicas diagnósticas y terapéuticas, que muchas veces se pueden hacer a distancia, sin que el paciente precise ingreso para hacer una monitorización o un seguimiento exhaustivo. La telemedicina, la consulta electrónica, la telemonitorización y otras técnicas en desarrollo, permitirán tener controlado al paciente como si realmente estuviera monitorizado en un centro hospitalario. Pero hay que ir más allá desarrollando fórmulas alternativas a la hospitalización convencional. Muchos pacientes crónicos que precisan ingreso, se pueden beneficiar más de la hospitalización ambulatoria que del ingreso en un centro hospitalario, pero en el momento actual no podemos atender toda la demanda del crónico en el medio ambulatorio, lo que significa que hay que potenciar los sistemas de hospitalización ambulatoria y otras fórmulas alternativas a la hospitalización convencional, siempre en estrecha colaboración con atención 
primaria. Este desarrollo requiere información, formación y cooperación por parte de la familia, voluntariado o individuos de la población formados para tal fin. El control de dichas formas alternativas de hospitalización, probablemente deba ser compartido entre atención primaria y hospitalizada y siempre con el protagonismo de la enfermería, que ya se está especializando en diferentes áreas, como ésta.

Uno de los retos a los que se enfrenta el sistema actual es la fragmentación organizativa, que hace que el paciente crónico y especialmente pluripatológico, sea abordado por diferentes especialistas que no son capaces de realizar un abordaje integral del paciente y por tanto, una estrategia integrada proactiva encaminada al mantenimiento funcional ${ }^{3}$. Precisamos romper esta fragmentación, lo que junto con los cambios demográficos y epidemiológicos, clínicos y de complejidad que se están produciendo en nuestra sociedad actual, suponen algunos de los principales retos a los que nos enfrentamos. Debemos intervenir pero centrándonos sobre todo en la salud poblacional y en la mejora de procesos para la calidad y productividad, porque el desarrollo científico y tecnológico, probablemente no supondrá un avance espectacular en los próximos años.

En definitiva, precisamos un modelo asistencial más proactivo y preventivo, una mejor gestión de las condiciones crónicas y de los pacientes complejos, una mayor focalización sobre la seguridad y la calidad en clínica. En este sentido el año pasado la National Center for Health Statistics americana publicó que la tercera causa de muerte en USA fueron los errores médicos, algo en lo que sin duda habrá que incidir. Igualmente precisamos formar a los pacientes y a la comunidad en el conocimiento de las enfermedades crónicas y a tener capacidad de decisión informada y compartida, mejorar la gestión de la salud poblacional, atacar desigualdades y lograr una mayor eficiencia del sistema.

Se estima que el 30\% de las actuaciones médicas diagnósticas y/o terapéuticas son ineficaces; tenemos que reconocerlas y evitarlas y en este sentido muchas sociedades científicas ya están proponiendo cuales son estas actuaciones que debemos reconocer y evitar.

En una sociedad que de nuevo parece que empieza a resurgir desde el punto de vista económico, se establecen diferencias socioeconómicas importantes, que harán más difícil la universalidad del sistema. Es preciso potenciar el apoyo social a los grupos más débiles y coordinarlo con los cuidados sanitarios, porque una estrategia poblacional dirigida al crónico que se base exclusivamente en aspectos sanitarios y no integre una política social adaptada, sin duda fracasará en sus resultados. Existen muchos sistemas de salud, casi tantos como países y todos distan mucho de ser ideales, por lo que tenemos que aprender algo de todos ellos y diseñar el nuestro adaptándolo a nuestras necesidades y a nuestra idiosincrasia.

En conclusión, podemos decir que es muy necesario el cambio del sistema sanitario público y que no existe un modelo ideal ni lo habrá, pero existen múltiples sistemas que se pueden adaptar al medio y a las circunstancias. El clínico sin duda, seguirá siendo protagonista, pero debe implicarse más en la gestión y en la innovación, porque es el clínico el que mejor conoce el problema y por tanto el que puede plantear las posibles soluciones. Debemos crear estructuras de trabajo que promuevan y capaciten la participación de los profesionales y diseñar redes para el aprendizaje y el intercambio del conocimiento, debemos aceptar que muchas respuestas están en la red y no en la propia organización sanitaria.

\section{Bibliografía}

1. Bortz W. J. A conceptual framework of frailty: a review. J Gerontol A Biol Sci Med Sci. 2002 May; 57: M283-8.

2. Bengoa R. Transforming health care: an approach to system-wide implementation. Int J Integr Care. 2013 Sep 25; 13:e039.

3. Ministry of Health, Social Services and Equality. EuropeanHealth Survey in Spain. 2009. Available from http://www.msssi.es/estadEstudios/estadisticas/EncuestaEuropea/home.htm.

4. National Center for Health Statistics. BMJ 2016. 